\title{
An Unsupervised Technique for Building Change Detection in Urban Area
}

\author{
Khaing Cho Moe \\ University of Computer Studies, \\ Yangon.
}

\author{
Myint Myint Sein \\ University of Computer Studies, \\ Yangon
}

\begin{abstract}
An unsupervised technique for building extraction in urban area is proposed, which is based on the use of morphological processing especially for building index. Extracting buildings is one of the most complex and challenging tasks as there exist a lot of inhomogeneity due to varying hierarchy. The variety of the type of the buildings and also the shapes of rooftops are very inconstant. This technique proposes a solution to the problem of automatic and unsupervised change detection of rooftop structures. The morphological operation is used to extract building feature with the use of modified morphological building index (MBI). Original Morphological Building Index (MBI) can extract interest building features only for multi-temporal high-resolution satellite image. But the proposed method is insensitive to the geometrical differences of buildings caused by different imaging conditions and is able to significantly reduce false alarms and also can detect upon low-resolution imagery. Image registration is applied after extracting building feature to overlay two images of the same scene. The effectiveness of the method is validated by comparing with MBI-based Change Vector Analysis (CVA) and Multivariate alteration detection (MAD) transformation.
\end{abstract}

\section{General Terms}

Image Processing and GIS

\section{Keywords}

Feature extraction, modified MBI, hue color histogram, image registration, RANSAC.

\section{INTRODUCTION}

Building change detection based on satellite imagery is becoming increasingly important for city monitoring, disaster assessment, and map database updating. Nowadays, Urban sprawl is a worldwide challenge. It is necessary to detect the land-cover/use changes occurring with urban sprawl and make plans for suitable development. Change detection is one of the main applications of remote sensing in [6]. Although urban land cover changes can be monitored by traditional ground survey procedures, now remote sensing sensors provide a cost-effective source of information for detecting important spatial patterns of land cover change over a large geographic area in a recurrent way.

The variety of change detection techniques has been researched extensively from a theoretical and practical aspect during the last decades. Bovolo [3] incorporated object-based image analysis into change vector analysis (CVA), by which the spatial features are combined with the spectral information for multi-temporal image analysis. Dalla Mura et al. [5] proposed to jointly use morphological filtering and the CVA algorithm for high-resolution image change detection in order to filter out commission errors caused by the geometrical differences in the multi-temporal images.
Jin and Davis [8] presented an automated building extraction strategy that simultaneously exploited structural, contextual, and spectral information. First, image structural information is extracted from the morphological profiles with a series of opening and closing operations. Second, shadows that are also removed from the morphological operations are used to provide reliable contextual information for buildings. Building may be surrounded by dense vegetation; they may have the same color as trees or trees may have colors other than green. Awrangjeb et al. [1] applies NDVI only and therefore cannot distinguish between a green building and a green tree. As a result, it fails to detect green buildings. Moreover, image quality may vary for the same scene even if images are captured by the same sensor, but at different dates and times.

Zitova and Flusser [2] presented image registration in multispectral satellite images is a crucial problem for remote sensing applications, and remains challenging because of the inherent nonlinearity. This is the process of overlaying two or more images of the same scene taken at different times, from different viewpoints and by different sensors. This makes better change detection result in accuracy rate.

Most of the changed buildings are detected in spite of some commission errors. X. Huang and L. Zhang [7] presented MBI extract building structures automatically but this method can be used for only high resolution satellite image because commission errors mainly result from the similar spectral attributes and the building areas of relatively low brightness.

In this paper, change detection of urban area by using modified MBI to extract building feature of satellite images from Google Earth is proposed. In our method, image registration technique is used to form mutual directional a registered pair image. Building structures are automatically extracted by the modified MBI from resulted registered pair image. This process is called feature extraction. The proposed method aims to automatically compare buildings and detect potential changes by a local building matching, rather than comparing the pixels or objects used in the traditional methods. According to the experiment result, this method is effective and efficient for both high and low quality satellite imagery. The paper is organized as follow, Section 2 and 3 present the overview of the proposed system and system methodology. In section 4 and 5, acknowledgement and the experimental result are described. Finally the conclusion is given in section 6 .

\section{OVERVIEW OF THE SYSTEM}

The overview of proposed urban change detection system is illustrated in figure 1 . The overview of proposed urban change detection system is surmised in figure 1. Firstly, the two year satellite images are grabbed into the system. These input images are classified into Red, Green and Blue using color segmentation and apply hue color histogram matching in order to calculate these two images input are same or different. If second images are not mostly change, the system 
shows there is no increasing building in these years and image registration process is applied to get registered images. Otherwise, the system goes on building extraction process. Then modified MBI is used to automatically extract features index of building from resulting registered image without using supervised learning. This method is effective for indication of buildings and will benefits to process low resolution images. Finally, change detection process is applied by using matching-based change rule and changed building area is displayed.

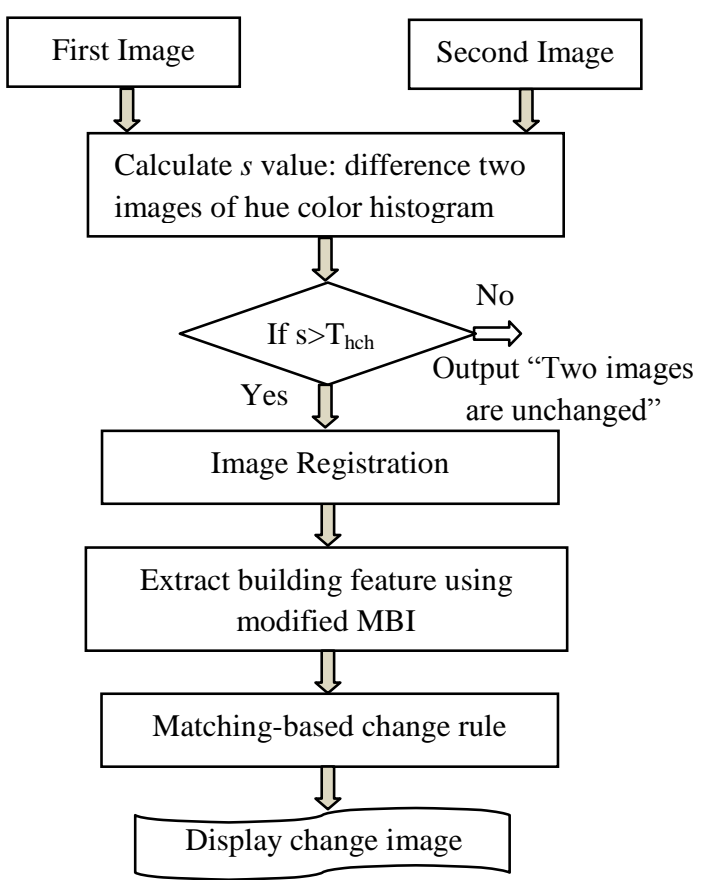

Figure 1: Overview of the proposed system

\section{SYSTEM METHODOLOGY}

\subsection{Hue Color Histogram Matching}

Firstly, input two images are tested by using combine approach of histogram equalization and hue color space to know these two images are same or not. This method overcomes unnecessary process of building change detection in unchanged or same urban area satellite image. Firstly, intensity value of input hue color images are calculated by the following $\mathrm{Eq}(1)$.

$$
s_{k}=T\left(h_{k}\right)
$$

for $\mathrm{k}=1,2, \ldots, \mathrm{L}$ where $s_{k}$ is the intensity value in the output image corresponding to the value $h_{k}$ in the input image of hue color component. The process continues or not is determined by the value of $s_{k}$. In my system, if histogram difference of two images is greater than threshold value $T_{e q}$, these two images are different. We generate many times for threshold value to get the best result using Otsu's method. Now, $T_{e q}=300$ is the most suitable for our system. Eq(2) specifies hue color component for intensity calculation.

$$
h=\left\{\begin{array}{cr}
\theta \quad \text { if } B \leq G \\
360-\theta & \text { if } B>G
\end{array}\right.
$$

where $B$ means blue axis and $G$ means green axis of HSI color space, $\theta$ is measured with respect to the red axis of HSI space.

\subsection{Image Registration}

An automatic image registration method has two broad categories: area-based and feature-based methods. Featurebased methods have been widely used in remote-sensing image registration. Feature-based image registration consists of five steps: preprocessing, feature selection, feature correspondence, transformation and resampling. These all steps are processed by automatically.

In this paper, the image registration process composes with the following steps:

$$
\begin{array}{ll}
\checkmark & \text { Feature point extraction (SURF method) } \\
\checkmark & \text { Find corresponding point pairs } \\
\checkmark & \text { Using RANSAC to remove outlier points } \\
\checkmark & \text { Apply affine transformation to obtain registered } \\
\text { image }
\end{array}
$$

The Speed-Up Robust Feature (SURF) method searches for matching points and then extracts all possible matching point pairs. It is also robust image detector and descriptor. The SURF detector is based on Hessian matrix which causes good performance and good accuracy.

After matching control point pairs have been identified, RANSAC algorithm is used to eliminate the outlier control point pairs of obtained a set of observed data. It can cope a large proportion of outliers in the input data. The advantages of using RANSAC outlier rejection method are it can robust to outliers and computational time grows quickly. And the number of hypothesis is sufficiently large that RANSAC gives very similar results.

\subsection{Morphological Building Index (MBI)}

Mathematical morphology is an effective tool for extracting image components that are useful in the representation and description of region features. Opening and closing are two commonly used operators, used to remove bright (opening) or dark (closing) details. Morphological operators are applied to an image with a set of a known shape, called a structural element (SE) described in [7]. Some key properties of the morphological transformation that are applied to the building extraction are summarized as follows.

- Reconstruction: The reconstruction filter is an important class of morphological filters that have been proven to be very useful for image processing.

- Granulometry: It describes the sizes and scales of objects in an image. Granulometrices have been introduced in remote sensing image classification of urban areas. The multi-scale morphological features are built based on the operators with increasing size of SE. Most of the existing morphological approaches referred to the disk-shaped SE. It is most convenient for our system.

The modified MBI is defined by describing the characteristic of building feature especially color of building roof and image intensity value. The system runs on low resolution satellite images so their resolution and brightness of intensity values are very low. In order to achieve this problem, modified MBI is proposed as the following steps:

\section{Step 1: Enhancement of Image}

The input low resolution registered image is transformed to high contrast image by applying with only red intensity value and stored as the brightness value which is computed by Eq. 3 


$$
g=T\left(f_{R}(x, y)\right)
$$

where $f_{R}(x, y)$ is a red color-space image, $s$ is the result of enhanced red band image. [9] Presented the maximum of multispectral bands for each pixels but it lessen brightness of input low resolution image to extract brightness value because it is only suitable in multispectral band of high resolution satellite image. Now our method gives for both high and low resolution of various satellite images as Google Earth.

\section{Step 2: Construction of MBI}

The spectral-structural characteristics of buildings (e.g., contrast, size and directionality) are represented using the Differential Morphological Profile (DMP). The construction of MBI contains three steps.

(i) White top-hat by Reconstruction can be computed by Eq. 4.

$$
W-T H(s)=g-\gamma_{b}^{r e}(s)
$$

where $\gamma_{b}^{r e}$ represents the opening-by-reconstruction of the brightness image, and $s$ indicates a flat and disk-shaped linear of structuring element, respectively.

(ii) Morphological Profiles (MP) of the white top-hat is defined as Eq. 5 and 6.

$$
\begin{aligned}
& M P_{W-T H}(s)=W-T H(s) \\
& M P_{W-T H}(s)=0
\end{aligned}
$$

(iii) Differential Morphological Profiles (DMP) of the white top-hat is calculated as Eq. 7.

$$
D M P_{W-T H}(s)=\left|M P_{W-T H}(s+\Delta s)-M P_{W-T H}(s)\right|
$$

where $\Delta \mathrm{s}$ is the interval of the profiles and $\mathrm{s}_{\min } \leq \mathrm{s} \leq \mathrm{s}_{\max }$

MBI is defined as the average of the DMPs of the white tophat profiles defined in eq. 8 and 9 since buildings have large local contrast in different directions within the range of the chosen scales. Thus

$$
\begin{gathered}
M B I=\frac{\sum_{s} D M P_{W-T H}(s)}{D \times S} \\
S=\left(\frac{S_{\max }-S_{\min }}{\Delta S}\right)+1
\end{gathered}
$$

where $\mathrm{D}$ and $\mathrm{S}$ indicate the numbers of the disk and scale of the profiles, respectively.

Step(3) Building extraction

$$
\begin{aligned}
& \operatorname{IF} \operatorname{MBI}(x) \geq t_{1} \text {, } \\
& \operatorname{THEN}_{\operatorname{map}}(x)=1 \\
& \operatorname{ELSE}_{\operatorname{map}}(x)=0
\end{aligned}
$$

where $\operatorname{MBI}(x)$ and $\operatorname{map}_{1}(x)$ indicate the value of $M B I$ and the initial label for pixel $x . \mathrm{t}_{1}$ is threshold value and set $\mathrm{t}_{1}=5$ for the best result for the system.

\section{Step 3: Postprocessing of MBI}

The building extraction process satisfies the following conditions and initial result of the building map is obtained by simply setting a threshold.

$$
\begin{aligned}
& \operatorname{IF} \operatorname{MBI}(x) \geq t 1,
\end{aligned}
$$

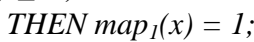

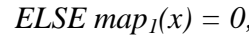

where $M B I(x)$ and $\operatorname{map}_{I}(x)$ indicate the value of $M B I$ and the result label for pixel $x$. $\mathrm{t}_{1}$ is threshold value and set $t_{l}=5$ for the best result for the system.

\subsection{Matched-based Change Rule}

After building only areas are extracted by modified MBI in two images, matched-based change rule is applied to get final change/increase building areas.

$$
\begin{aligned}
& \text { If } \overline{\operatorname{map}}_{1}(i) \cap \overline{\operatorname{map}}_{2}(i) \\
& \text { then } C(i)=0 . \\
& \text { elseif } \overline{\operatorname{map}}_{1}(i) \cap \operatorname{map}_{2}(i) \\
& \text { then } C(i)=1 . \\
& \text { elseif } \operatorname{map}_{1}(i) \cap \overline{\operatorname{map}}_{2}(i) \\
& \text { then } C(i)=0 . \\
& \text { else } \operatorname{map}_{1}(i) \cap \operatorname{map}_{2}(i) \\
& \text { then } C(i)=0 .
\end{aligned}
$$$$
\text { end } \quad i \in 1,2,3, \ldots, N
$$

where $\operatorname{map}_{1}(i)$ and $\operatorname{map}_{2}(i)$ are the output value( $(0$ and 1$)$ of modified MBI method. ' 0 ' means no building and ' 1 ' means building. The $i$ is the same pixel of first and second images and $\mathrm{N}$ is the number of pairs of the corresponding building objects where $C(i)$ represents whether the object $i$ is changed, with 0 and 1 for non-change and change, respectively.

\section{EXPERIMENTAL RESULT}

The experiments are conducted on Google Earth satellite images of Kyi Myin Daing Township, Yangon, Myanmar. Figure 2(a) and (b) show Original satellite input image of 2003 and 2010.
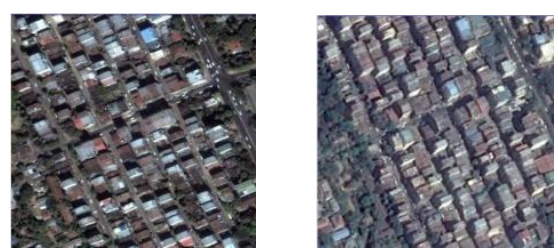

Figure 2: Original input image of (a) 2003 and (b) 2010.

Figure 3 compares two images to know they are same or different in building area using hue color histogram matching. This can be seen that the two images of pixels value difference are greater than defined threshold value. In this stage, pre-processing step can achieve better performance and reduce time consuming.

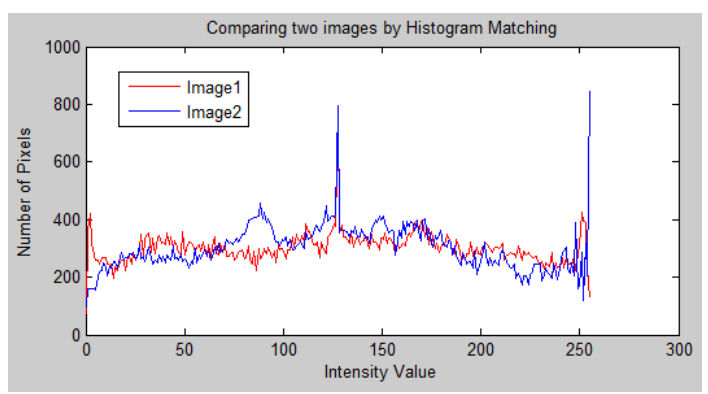

Figure 3: Hue color histogram matching

Now pixel value difference of two images are greater than pre-defined threshold value, the image registration process continues to get registered images for better building change 
detection. Figure 4 shows the result of image registration using SURF and RANSAC.

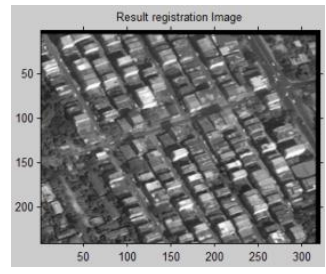

Fig: Output registered image of year 2003 and 2010

Then modified MBI is applied to extract building feature as shown in figure 5 . The input images are divided into four parts and process them by modified MBI method. Then, these all parts are combined to get one image as shown in figure 6 in which white area means building areas and black area indicates open space areas that have no buildings and can build anymore.

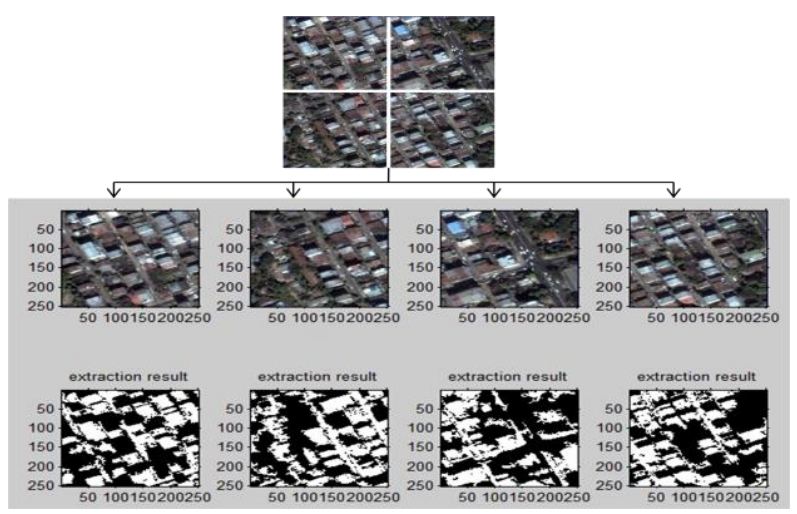

(a)
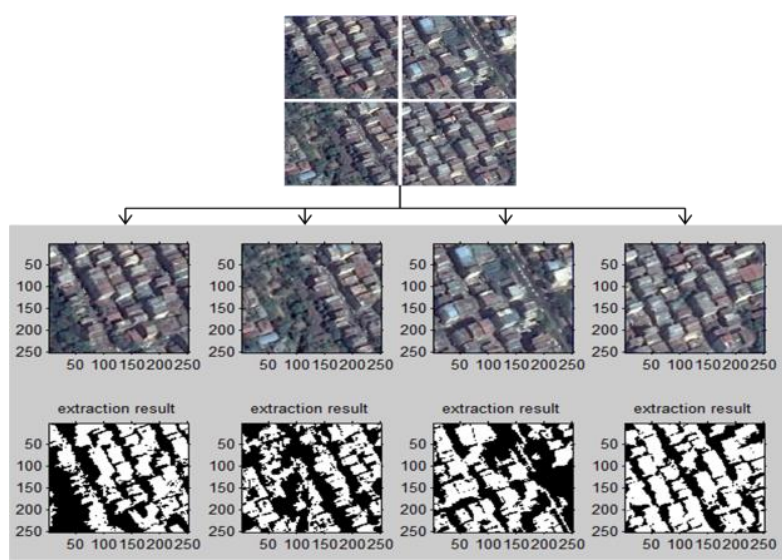

(b)

Figure 5: building extraction result of (a) 2003 and (b) 2010images.

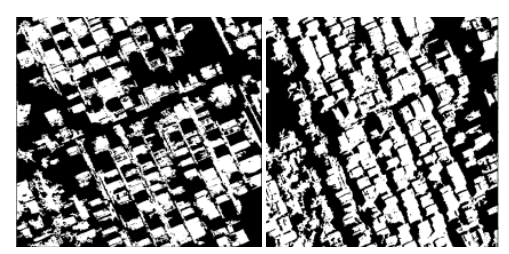

(a)

(b)

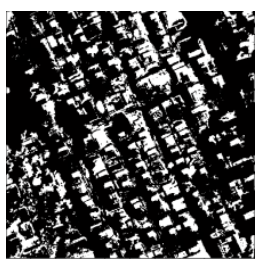

Figure 7: Output of proposed change detection result

Final change detection result obtained from using only matched-based change rule is shown in figure 7 in which the black areas are changed/increase building and others are without changed area between these two year and most changes have been correctly detected, with a high accuracy of $97 \%$. However, several changed area are still missed.

The performance of the proposed modified MBI and matching-based change rule is compared with change detection approach of MBI-based CVA in [9] and Multivariate alteration detection (MAD) transformation in [4]. MBI-based CVA focus on building change detection. The spectral bands are replaced by the multi-temporal MBI feature images for CVA. It contains the salt-and-pepper effects of pixel-based change detection approaches. Multivariate alteration detection (MAD) transformation approach gives good detection results for only simplified data but the variety of buildings shape and the heterogeneity of roof types limited the effectiveness of the proposed methodology.

Table 1: Accuracy assessment of the proposed method

\begin{tabular}{|l|l|l|l|}
\hline $\begin{array}{l}\text { Quantitative } \\
\text { measures }\end{array}$ & MBI-based & MAD & Proposed \\
& CVA & & Method \\
\hline Correctness & $81.75 \%$ & $77.43 \%$ & $97.87 \%$ \\
\hline Completeness & $74.79 \%$ & $88.03 \%$ & $92.45 \%$ \\
\hline Quality & $64.09 \%$ & $66.32 \%$ & $89.07 \%$ \\
\hline
\end{tabular}

To test the performance of the proposed system, we first implement change detection of MBI-based CVA and MAD approach. The evaluation of change detection was accomplished using evaluation measures (completeness, correctness, quality).

$$
\begin{aligned}
& \text { Correctness }=\frac{T P}{T P+F P} \\
& \text { Completeness }=\frac{T P}{T P+F N} \\
& \text { Quality }=\frac{T P}{T P+F P+F N}
\end{aligned}
$$

where TP (True Positive) and FP (False Positive) are the numbers of changed pixels in the result, but changed and unchanged in the reference image, respectively. FN (False Negative) is the number of changed pixels in the reference image, but detected as unchanged in the result. Table 1 illustrates the accuracy assessment in three methods of change detection. A comparison between the proposed method and several recently developed change detection methods revealed that the proposed method reduced the number of commission and omission errors significantly.

Figure 6: Combine image of fig 4: (a) 2003 and (b) 2010 images 


\section{ACKNOWLEDGMENTS}

The author thankfully acknowledges Kazuaki Iwamura, Senior Engineer, Smart Information Systems Division, Information and Telecommunication Systems Company, Hitachi, Ltd., and Eiichiro UMINO, Lead Engineer, GIS Department, Hitachi Solutions, Ltd., for giving us two week training course for GIS/GeoMation in our university and his technical guidance.

\section{CONCLUSION}

In this paper, we have presented a building change detection method from satellite urban images, which is able to solve various intensity colors of building roof and uncertainly of building structure with the help of modified MBI result. It is not an easy task to automatically extract buildings without any supervised learning. But, it can be overcome by modified MBI and it is effective method for indication of buildings for even low quality resolution images. The qualitative and quantitative analysis of the changed detection result validates the effectiveness of the proposed system. The system also eases the constructor to find how area is free or already built and provides the free space of open area to build.

\section{REFERENCES}

[1] Awrangjeb, M., M. Ravanbakhsh, and C.S. Fraser, "Automatic detection of residential buildings using lidar data and multispectral imagery," ISPRS Journal of Photogrammetry and Remote Sensing, 65(5):457-467. 2010.

[2] B. Zitova and J.Flusser, "Image registration methods: A survey," Image Vis. Comput., Vol. 21, No, 11, pp. 977 1000, 2004.
[3] F. Bovolo, "A multilevel parcel-based approach to change detection in very high resolution multitemporal images," IEEE Geosci. Remote Sens. Lett., vol. 6, no. 1, pp. 3337, Jan. 2009

[4] G. Doxani, K. Karantzalos, M.Tsakiri, "Automatic Change Detection in Urban Areas under A Scale-space, object-oriented classification framework," Oct 22, 2009.

[5] M. Dalla Mura, "An unsupervised technique based on morphological filters for change detection in very high resolution images," IEEE Geosci. Remote Sens. Lett., vol. 5, no. 3, pp. 433-437, Jul. 2008.

[6] R. J. Radke, S. Andra, O. Al-Kofahi, and B. Roysam, "Image change detection algorithms: Systematic survey," IEEE Trans: Image Process., vol. 14, no. 3, pp. 294-307, Aug. 2005.

[7] X. Huang and L.Zhang, "A multidirectional and multiscale morphological index for automatic building extraction from multispectral GeoEye-1 imagery," Photogramm, Eng, Remote Sens., vol. 5, no. 1, pp. 161172, Feb, 2012.

[8] X. Jin and C.H. Davis, "Automated building extraction from high resolution satellite imagery in urban areas using structural, contextual, and spectral information," EURASIP J.Appl. Signal Process.,vol. 14, pp. 21962206, Jan. 2005.

[9] Y. Tang, X. Huang and L. Zhang, "Fault-Tolerant building change detection from urban high-resolution remote rensing imagery," IEEE Geosci. Remote Sens. Lett, vol.10, No.5, Sept 2013. 